\title{
Syntax and semantics of simple paracomplete logics ${ }^{1}$
}

\author{
Vladimir M. Popov, Vasiliy O. Shangin
}

\begin{abstract}
For an arbitrary fixed element $\beta$ in $\{1,2,3, \ldots \omega\}$ both a sequent calculus and a natural deduction calculus which axiomatise simple paracomplete logic $\mathrm{I}_{2, \beta}$ are built. Additionally, a valuation semantic which is adequate to $\operatorname{logic} \mathrm{I}_{2, \beta}$ is constructed. For an arbitrary fixed element $\gamma$ in $\{1,2,3, \ldots\}$ a cortege semantic which is adequate to logic $\mathrm{I}_{2, \gamma}$ is described. A number of results obtainable with the axiomatisations and semantics in question are formulated.
\end{abstract}

Keywords: paracomplete logic, paraconsistent logic, cortege semantics, valuation semantics, sequent calculus, natural deduction calculus

We study logics $\mathrm{I}_{2,1}, \mathrm{I}_{2,2}, \mathrm{I}_{2,3}, \ldots \mathrm{I}_{2, \omega}$ presented in [8]. These logics are paracomplete counterparts of paraconsistent logics $\mathrm{I}_{1,1}, \mathrm{I}_{1,2}, \mathrm{I}_{1,3}$, $\ldots \mathrm{I}_{1, \omega}$ from [7]. In the paper, (a) simple paracomplete logics $\mathrm{I}_{2,1}$, $\mathrm{I}_{2,2}, \mathrm{I}_{2,3}, \ldots \mathrm{I}_{2, \omega}$ are defined (see [8]); these logics form (in the order indicated above) a strictly decreasing (in terms of the set-theoretic inclusion) sequence of logics, (b) for any $j$ in $\{0,1,2,3, \ldots \omega\}$ both a sequent calculus $\mathrm{GI}_{2, j}$ (see [10]) and a natural deduction calculus $\mathrm{NI}_{2, j}$ which axiomatise logic $\mathrm{I}_{2, j}$ are formulated, (c) for any $j$ in $\{1,2,3, \ldots \omega\}$, we propose a valuation semantics for logic $I_{2, j}$ (see [9]), (d) for any $j$ in $\{1,2,3, \ldots\}$, we propose a cortege semantics for logic $\mathrm{I}_{2, j}$ (see [9]). Below there are some results obtained with the semantics and calculi in question.

The language $L$ of each logic in the paper is a standard propositional language with the following alphabet: $\{\&, \vee$,

\footnotetext{
${ }^{1}$ The paper is supported by Russian Foundation for Humanities, project №10-03-00570a and project №13-03-00088a (both authors).
} 
$\left.\supset, \neg,(),, p_{1}, p_{2}, p_{3}, \ldots\right\}$. As it is expected, \&, $\vee, \supset$ are binary logical connectives in $L, \neg$ is a unary logical connective in $L$, brackets $($,$) are technical symbols in L$ and $p_{1}, p_{2}, p_{3}, \ldots$ are propositional variables in $L$. A definition of $L$-formula is as usual. Below, we say 'formula' instead of ' $L$-formula' only and adopt the convention on omitting brackets as in [4]. A formula is said to be quasi-elemental iff no logical connective in $L$ other than $\neg$ occurs in it. A length of a formula $A$ is, traditionally, said to be the number of all occurrences of the logical connectives in $L$ in $A$. We denote the rule of modus ponens in $L$ by MP and the rule of substitution of a formula into a formula instead of a propositional variable in $L$ by Sub. A logic is said to be a non-empty set of formulas closed under MP and Sub. A theory for logic $\mathbf{L}$ is said to be a set of formulas including logic $\mathbf{L}$ and closed under MP. It is understood that the set of all formulas is both a logic and a theory for any logic. The set of all formulas is said to be a trivial theory. A complete theory for logic $\mathbf{L}$ is said to be a theory $\mathrm{T}$ for logic $\mathbf{L}$ such that, for some formula $A$, $A \in \mathrm{T}$ or $\neg A \in \mathrm{T}$. A paracomplete theory for logic $\mathbf{L}$ is said to be a theory $\mathrm{T}$ for $\operatorname{logic} \mathbf{L}$ such that $\mathrm{T}$ is not a complete theory and any complete theory for logic $\mathbf{L}$, which includes $\mathrm{T}$, is a trivial theory. A paracomplete logic is said to be a logic $\mathbf{L}$ such that there exists a paracomplete theory for logic $\mathbf{L}$. Simple paracomplete logic is said to be a paracomplete logic $\mathbf{L}$ such that for any paracomplete theory $\mathrm{T}$ for logic $\mathbf{L}$ holds true: there exists a quasi-elemental formula $A$ such that neither $A$, nor $\neg A$ belongs to T.

Let us agree that anywhere in the paper: $\alpha$ is an arbitrary element in $\{0,1,2,3, \ldots \omega\}, \beta$ is an arbitrary element in $\{1,2,3, \ldots \omega\}, \gamma$ is an arbitrary element in $\{1,2,3, \ldots\}$. We define calculus $\mathrm{HI}_{2, \alpha}$. This calculus is Hilbert-type calculi, the language of $\mathrm{HI}_{2, \alpha}$ is $L$. $\mathrm{HI}_{2, \alpha}$ has $\mathrm{MP}$ as the only rule of inference. The notion of a derivation in $\mathrm{HI}_{2, \alpha}$ (of a proof in $\mathrm{HI}_{2, \alpha}$, in particular) is defined as usual; and for $\mathrm{HI}_{2, \alpha}$, both notion of a formula derivable from the set of formulas in this calculus and a notion of a formula provable in this calculus are defined as usual. Now we only need to define the set of axioms of $\mathrm{HI}_{2, \alpha}$.

A formula belongs to the set of axioms of calculus $\mathrm{HI}_{2, \alpha}$ iff it is one of the following forms (hereafter, $A, B, C$ denote formulas): 
(I) $(A \supset B) \supset((B \supset C) \supset(A \supset C))$, (II) $A \supset(A \vee B)$, (III) $B \supset(A \vee B),(\mathrm{IV})(A \supset C) \supset((B \supset C) \supset((A \vee B) \supset C))$, $(\mathrm{V})(A \& B) \supset A,(\mathrm{VI})(A \& B) \supset B,(\mathrm{VII})(C \supset A) \supset((C \supset B) \supset$ $(C \supset(A \& B)))$, (VIII) $(A \supset(B \supset C)) \supset((A \& B) \supset C)$, (IX) $((A \& B) \supset C) \supset(A \supset(B \supset C)),(\mathrm{X})((A \supset B) \supset A) \supset A,(\mathrm{XI}, \alpha)$ $(E \supset \neg(B \supset B)) \supset \neg E$, where $E$ is formula which is not a quasielemental formula of a length less than $\alpha$, (XII) $\neg A \supset(A \supset B)$.

Let us agree that, for any $j$ in $\{0,1,2,3, \ldots \omega\}, \mathrm{I}_{2, j}$ is the set of formulas provable in $\mathrm{HI}_{2, j}$.

The following theorems 1 and 2 are shown.

Theorem 1. Sets $I_{2,0}, I_{2,1}, I_{2,2}, I_{2,3}, \ldots I_{2, \omega}$ are logics, and, for any $k$ and $l$ in $\{0,1,2,3, \ldots \omega\}$, if $k<l$, then $I_{2, l} \subseteq I_{2, k}$.

TheOREM 2. Logic $I_{2,0}$ is the set of the classical tautologies in $L$.

Let us establish connections between logics $\mathrm{I}_{2,1}, \mathrm{I}_{2,2}, \mathrm{I}_{2,3}, \ldots \mathrm{I}_{2, \omega}$ and logic $\mathrm{I}_{2,0}$ (that is, the classical propositional logic in $L$ ).

Let $\varphi$ be a mapping of the set of all formulas into itself satisfying the following conditions: (1) $\varphi(p)$ is not a quasi-elemental formula, for any propositional variable $p$ in $L,(2)$ for any propositional variable $p$ in $L$, formulas $p \supset \varphi(p)$ and $\varphi(p) \supset p$ belong to logic $\mathrm{I}_{2,0}$, (3) $\varphi(B \circ C)=\varphi(B) \circ \varphi(C)$, for any formulas $B, C$ and for any binary logical connective $\circ$ in $L,(4) \varphi(\neg B)=\neg \varphi(B)$, for any formula $B$.

Following these conditions, theorem 3 is shown.

Theorem 3. For any $j$ in $\{1,2,3, \ldots \omega\}$ and for any formula $A$ : $A \in I_{2,0}$ iff $\varphi(A) \in I_{2, j}$.

Let now $\psi$ be such a mapping the set of all formulas into itself satisfying the following conditions: (1) $\psi(p)=p$, for any propositional variable $p$ in $L,(2) \psi(B \circ C)=\psi(B) \circ \psi(C)$, for any formulas $B, C$ and for any binary logical connective $\circ$ in $L$, (3) $\psi(\neg B)=\psi(B) \supset \neg\left(p_{1} \supset p_{1}\right)$, for any formula $B$.

Following these conditions, theorem 4 is shown.

Theorem 4. For any $j$ in $\{1,2,3, \ldots \omega\}$ and for any formula $A$ : $A \in I_{2,0}$ iff $\psi(A) \in I_{2, j}$.

Let us now show a method to build up a sequent calculus $\mathrm{GI}_{2, \beta}$ which axiomatises logic $\mathrm{I}_{2, \beta}$. Calculus $\mathrm{GI}_{2, \beta}$ (see [10]) is a Gentzen- 
type sequent calculus. Sequents are of the form $\Gamma \rightarrow \Delta$ (hereafter, $\Gamma, \Delta, \Sigma$ and $\Theta$ denote finite sequences of formulas). The set of basic sequents of $\mathrm{GI}_{2, \beta}$ is the set of all sequents of the form $A \rightarrow A$. The only rules of $\mathrm{GI}_{2, \beta}$ are the rules $\mathrm{R} 1-\mathrm{R} 15, \mathrm{R} 16(\beta), \mathrm{R} 17$ listed below.

$$
\begin{aligned}
& \frac{\Gamma, A, B, \Delta \rightarrow \Theta}{\Gamma, B, A, \Delta \rightarrow \Theta} \mathrm{R} 1, \frac{\Gamma \rightarrow \Delta, A, B, \Theta}{\Gamma \rightarrow \Delta, B, A, \Theta} \mathrm{R} 2, \frac{A, A, \Gamma \rightarrow \Theta}{A, \Gamma \rightarrow \Theta} \mathrm{R} 3, \\
& \frac{\Gamma \rightarrow \Theta, A, A}{\Gamma \rightarrow \Theta, A} \mathrm{R} 4, \quad \frac{\Gamma \rightarrow \Theta}{A, \Gamma \rightarrow \Theta} \mathrm{R} 5, \quad \frac{\Gamma \rightarrow \Theta}{\Gamma \rightarrow \Theta, A} \mathrm{R} 6, \\
& \frac{\Gamma \rightarrow \Delta, A \quad B, \Sigma \rightarrow \Theta}{A \supset B, \Gamma, \Sigma \rightarrow \Delta, \Theta} \mathrm{R} 7, \frac{A, \Gamma \rightarrow \Theta, B}{\Gamma \rightarrow \Theta, A \supset B} \mathrm{R} 8 \\
& \frac{A, \Gamma \rightarrow \Theta}{A \& B, \Gamma \rightarrow \Theta} \mathrm{R} 9, \quad \frac{A, \Gamma \rightarrow \Theta}{B \& A, \Gamma \rightarrow \Theta} \mathrm{R} 10, \quad \frac{\Gamma \rightarrow \Theta, A \quad \Gamma \rightarrow \Theta, B}{\Gamma \rightarrow \Theta, A \& B} \mathrm{R} 11, \\
& \frac{\Gamma \rightarrow \Theta, A}{\Gamma \rightarrow \Theta, A \vee B} \mathrm{R} 12, \frac{\Gamma \rightarrow \Theta, A}{\Gamma \rightarrow \Theta, B \vee A} \mathrm{R} 13, \frac{A, \Gamma \rightarrow \Theta \quad B, \Gamma \rightarrow \Theta}{A \vee B, \Gamma \rightarrow \Theta} \mathrm{R} 14, \\
& \frac{\Gamma \rightarrow \Theta, A}{\neg A, \Gamma \rightarrow \Theta} \mathrm{R} 15 \\
& \begin{array}{l}
\frac{E, \Gamma \rightarrow \Theta}{\Gamma \rightarrow \Theta, \neg E} \mathrm{R} 16(\beta), \text { where } E \text { is a formula which is not a quasi-elemental } \\
\text { formula of a length less than } \beta,
\end{array} \\
& \frac{\Gamma \rightarrow \Delta, A \quad A, \Sigma \rightarrow \Theta}{\Gamma, \Sigma \rightarrow \Delta, \Theta} \mathrm{R} 17 \text { (cut rule) }
\end{aligned}
$$

A derivation in calculus $\mathrm{GI}_{2, \beta}$ is defined in a standard sequent calculus fashion. The definition of a sequent provable in $\mathrm{GI}_{2, \beta}$ is as usual. The cut-elimination theorem is shown (by Gentzen's method presented in [3]) to be valid in $\mathrm{GI}_{2, \beta}$.

The following theorem 5 is shown.

Theorem 5. For any $j$ in $\{1,2,3, \ldots \omega\}$ and for any formula $A$ : $A \in I_{2, j}$ iff a sequent $\rightarrow A$ is provable in $G I_{2, j}$.

Let us now show a method to build up a Fitch-style natural deduction calculus $\mathrm{NI}_{2, \beta}$ which axiomatises logic $\mathrm{I}_{2, \beta}$.

The set of $\mathrm{NI}_{2, \beta}$-rules is as follows, where $[A] C$ denotes a derivation of a formula $C$ from a formula $A$.

$$
\frac{C \& C_{1}}{C} \&_{e l 1} \quad \frac{C \& C_{1}}{C_{1}} \&_{e l 2} \quad \frac{C, C_{1}}{C \& C_{1}} \&_{i n}
$$




$$
\begin{aligned}
& \frac{C \vee C_{1},[C] C_{2}\left[C_{1}\right] C_{2}}{C_{2}} \vee_{e l} \quad \frac{C}{C \vee C_{1}} \vee_{i n 1} \quad \frac{C_{1}}{C \vee C_{1}} \vee_{i n 2} \\
& \frac{C \supset C_{1}, C}{C_{1}} \supset_{e l} \quad \frac{[C] C_{1}}{C \supset C_{1}} \supset_{i n} \quad \frac{[A \supset B] A}{A} \supset_{p} \\
& \frac{[E] \neg(C \supset C)}{\neg E} \neg \operatorname{in} 1(\beta) \text {, where } E \text { is a formula which is not a quasi- } \\
& \frac{\neg C_{1}, C_{1}}{C} \neg \neg_{\text {in } 2}
\end{aligned}
$$

A derivation in $\mathrm{NI}_{2, \beta}$ is defined in a standard natural deduction calculus fashion.

The following theorem 6 is shown.

Theorem 6. For any $j$ in $\{1,2,3, \ldots \omega\}$ and for any formula $A$ : $A \in I_{2, j}$ iff $A$ is provable in $N I_{2, j}$.

The proof search procedures which were proposed to the classical and a variety of non-classical logics are applicable $[1,2]$.

Let us construct $I_{2, \beta}$-valuation semantics for $I_{2, \beta}$. By $Q_{\beta}$ we denote the set of all quasi-elemental formulas of a length less or equal to $\beta$. By $\mathrm{I}_{2, \beta}$-valuation we mean any mapping $v$ set $\mathrm{Q}_{\beta}$ into the set $\{0,1\}$ such that, for any quasi-elemental formula $e$ of a length less than $\beta$, if $v(e)=1$, then $v(\neg e)=0$. Let Form denote the set of all formulas and let $\mathrm{Val}_{2, \beta}$ denote the set of all $\mathrm{I}_{2, \beta}$-valuations. It can be shown there exists a unique mapping (denoted by $\xi_{2, \beta}$ ) satisfying the following six conditions: (1) $\xi_{2, \beta}$ is a mapping a Cartesian product Form $\times \operatorname{Val}_{2, \beta}$ into the set $\{1,0\},(2)$ for any quasi-elemental formula $Y$ in $\mathrm{Q}_{\beta}$ and any $\mathrm{I}_{2, \beta}$-valuation $v: \xi_{2, \beta}(Y, v)=v(Y)$, (3) for any formulas $A, B$ and any $\mathrm{I}_{2, \beta}$-valuation $v: \xi_{2, \beta}(A \& B, v)=1$ iff $\xi_{2, \beta}(A)=1$ and $\xi_{2, \beta}(B)=1$, (4) for any formulas $A, B$ and any $\mathrm{I}_{2, \beta^{-}}$ valuation $v: \xi_{2, \beta}(A \vee B, v)=1$ iff $\xi_{2, \beta}(A)=1$ or $\xi_{2, \beta}(B)=1$, (5) for any formulas $A, B$ and any $\mathrm{I}_{2, \beta}$-valuation $v: \xi_{2, \beta}(A \supset B, v)=1$ iff $\xi_{2, \beta}(A)=0$ or $\xi_{2, \beta}(B)=1$, (6) for any formula $A$ which is not a quasi-elemental formula of a length less than $\beta$, and for any $\mathrm{I}_{2, \beta^{-}}$ valuation $v$ : $\xi_{2, \beta}(\neg A, v)=1$ iff $\xi_{2, \beta}(A, v)=0$. A formula $A$ is said to be $\mathrm{I}_{2, \beta}$-valid iff for any $\mathrm{I}_{2, \beta}$-valuation $v, \xi_{2, \beta}(A, v)=1$.

The following theorems 7 and 8 are shown.

Theorem 7. For any $j$ in $\{1,2,3, \ldots \omega\}$, for any formula $A$, for any set $\Gamma$ of formulas: formula $A$ is derivable from $\Gamma$ in $H I_{2, j}$ iff for 
any $I_{2, j}$-valuation $v$, if for any formula $B$ in $\Gamma, \xi_{2, j}(B, v)=1$, then $\xi_{2, j}(A, v)=1$.

Theorem 8. For any $j$ in $\{1,2,3, \ldots \omega\}$ and for any formula $A$, $A \in I_{2, j}$ iff formula $A$ is $I_{2, j}$-valid.

It should be noted that the proposed $\mathrm{I}_{2, \beta}$-valuation semantics is consistent to the requirements, which, in our point of view, N.A. Vasiliev considers to be necessary in [11]: (1) no proposition cannot be true and false at once, (2) in general case, a value of the proposition that is a negation of a proposition $P$, is not determined by the value of $P$.

Let us construct $\mathrm{I}_{2, \gamma}$-cortege semantics for $\mathrm{I}_{2, \gamma}$. By $\mathrm{I}_{2, \gamma}$-cortege we mean an ordered $\gamma+1$-tuplet of elements of the set $\{1,0\}$ such that for any two neighboring members of this ordered $\gamma+1$-tuplet, at least one of them is 0 . By a designated $\mathrm{I}_{2, \gamma}$-cortege we mean $\mathrm{I}_{2, \gamma}$-cortege, where the first member is $1 . \mathrm{By} \mathrm{S}_{2, \gamma}$ we denote the set of all $\mathrm{I}_{2, \gamma}$-corteges and by $\mathrm{D}_{2, \gamma}$ we denote the set of all designated $\mathrm{I}_{2, \gamma}$-corteges. By a normal $\mathrm{I}_{2, \gamma}$-cortege we mean $\mathrm{I}_{2, \gamma}$-cortege such that any two neighboring members of this $\mathrm{I}_{2, \gamma}$-cortege are different. By a single $\mathrm{I}_{2, \gamma}$-cortege we mean a normal $\mathrm{I}_{2, \gamma}$-cortege such that the first member of it is 1 . By a zero $\mathrm{I}_{2, \gamma}$-cortege we mean a normal $\mathrm{I}_{2, \gamma}$-cortege such that the first member of it is 0 .

It is clear that there exists a unique single $\mathrm{I}_{2, \gamma}$-cortege (denoted by $\mathbf{1}_{\gamma}$ ) and there exists a unique zero $\mathrm{I}_{2, \gamma}$-cortege (denoted by $\mathbf{0}_{\gamma}$ ). It can be shown that there exists a unique binary operation on $\mathrm{S}_{2, \gamma}$ (denoted by $\&_{2, \gamma}$ ) satisfying the following condition, for any $X, Y$ in $\mathrm{S}_{2, \gamma}$ : if the first member of $\mathrm{I}_{2, \gamma}$-cortege $X$ is 1 and the first member of $\mathrm{I}_{2, \gamma}$-cortege $Y$ is 1 then $X \&_{2, \gamma} Y$ is $\mathbf{1}_{\gamma}$; otherwise, $X \&_{2, \gamma} Y$ is $\mathbf{0}_{\gamma}$. It can be shown that there exists a unique binary operation on $\mathrm{S}_{2, \gamma}$ (denoted by $\vee_{2, \gamma}$ ) satisfying the following condition, for any $X$ and $Y$ in $\mathrm{S}_{2, \gamma}$ : if the first member of $\mathrm{I}_{2, \gamma}$-cortege $X$ is 1 or the first member of $\mathrm{I}_{2, \gamma}$-cortege $Y$ is 1 then $X \vee_{2, \gamma} Y$ is $\mathbf{1}_{\gamma}$; otherwise, $X \vee_{2, \gamma} Y$ is $\mathbf{0}_{\gamma}$. It can be shown that there exists a unique binary operation on $\mathrm{S}_{2, \gamma}$ (denoted by $\supset_{2, \gamma}$ ) satisfying the following condition, for any $X$ and $Y$ in $\mathrm{S}_{2, \gamma}$ : if the first member of $\mathrm{I}_{2, \gamma}$-cortege $X$ is 0 or the first member of $\mathrm{I}_{2, \gamma}$-cortege $Y$ is 1 then $X \supset_{2, \gamma} Y$ is $\mathbf{1}_{\gamma}$; otherwise, $X \supset_{2, \gamma} Y$ is $\mathbf{0}_{\gamma}$. It can be shown that there exists a unique unary 
operation on $\mathrm{S}_{2, \gamma}$ (denoted by $\neg 2, \gamma$ ) satisfying the following condition, for any $\mathrm{I}_{2, \gamma}$-cortege $\left\langle x_{1}, x_{2}, \ldots, x_{\gamma}, x_{\gamma+1}\right\rangle$ : if $x_{\gamma+1}$ is 1 then $\left.\neg 2, \gamma\left(<x_{1}, x_{2}, \ldots, x_{\gamma}, x_{\gamma+1}>\right)=<x_{2}, \ldots, x_{\gamma}, x_{\gamma+1}, 0\right\rangle$ and if, if $x_{\gamma+1}$ is 0 , then $\neg 2, \gamma\left(<x_{1}, x_{2}, \ldots, x_{\gamma}, x_{\gamma+1}>\right)=$ $<x_{2}, \ldots, x_{\gamma}, x_{\gamma+1}, 1>$.

It is clear that $<\mathrm{S}_{2, \gamma}, \mathrm{D}_{2, \gamma}, \&_{2, \gamma}, \vee_{2, \gamma}, \supset_{2, \gamma}, \neg_{2, \gamma}>$ is a logical matrix. This logical matrix (denoted by $\mathrm{M}_{2, \gamma}$ ) is said to be $\mathrm{I}_{2, \gamma}$-matrix. $\mathrm{M}_{2, \gamma}$-valuation is said to be a mapping the set of all propositional variables in $L$ into $\mathrm{S}_{2, \gamma}$. The set of all $\mathrm{M}_{2, \gamma^{-}}$ valuations is denoted by $\mathrm{ValM}_{2, \gamma}$. It can be shown that there exists a unique mapping (denoted by $\xi \mathrm{M}_{2, \gamma}$ ) satisfying the following conditions: (1) $\xi \mathrm{M}_{2, \gamma}$ is a mapping a Cartesian product Form $\mathrm{x}$ ValM $_{2, \gamma}$ into the set $S_{2, \gamma}$, (2) for any propositional variable $p$ in $L$ and for any $\mathrm{M}_{2, \gamma}$-valuation $w, \xi \mathrm{M}_{2, \gamma}(p, w)=w(p)$, (3) for any formulas $A, B$ and for any $\mathrm{M}_{2, \gamma}$-valuation $w, \xi \mathrm{M}_{2, \gamma}(A \& B, w)=$ $\xi \mathrm{M}_{2, \gamma}(A, w) \&_{2, \gamma} \xi \mathrm{M}_{2, \gamma}(B, w)$, (4) for any formulas $A, B$ and for any $\mathrm{M}_{2, \gamma}$-valuation $w, \xi \mathrm{M}_{2, \gamma}(A \vee B, w)=\xi \mathrm{M}_{2, \gamma}(A, w) \vee_{2, \gamma} \xi \mathrm{M}_{2, \gamma}(B, w)$, (5) for any formulas $A, B$ and for any $\mathrm{M}_{2, \gamma}$-valuation $w, \xi \mathrm{M}_{2, \gamma}(A \supset$ $B, w)=\xi \mathrm{M}_{2, \gamma}(A, w) \supset_{2, \gamma} \xi \mathrm{M}_{2, \gamma}(B, w),(6)$ for any formula $A$ and for any $\mathrm{M}_{2, \gamma}$-valuation $w, \xi \mathrm{M}_{2, \gamma}(\neg A, w)=\neg 2, \gamma \xi \mathrm{M}_{2, \gamma}(A, w)$.

A formula $A$ is said to be $\mathrm{M}_{2, \gamma}$-valid iff for any $\mathrm{M}_{2, \gamma}$-valuation $w$, $\xi \mathrm{M}_{2, \gamma}(A, w) \in \mathrm{D}_{2, \gamma}$.

The following theorems 9-11 are shown.

Theorem 9. For any $j$ in $\{1,2,3, \ldots\}$, for any formula $A$ and for any set $\Gamma$ of formulas, formula $A$ is derivable from $\Gamma$ in $\mathrm{HI}_{2, j}$ iff for any $M_{2, j}$-valuation $w$, if for any formula $B$ from $\Gamma, \xi M_{2, j}(B, w) \in$ $D_{1, j}$ then $\xi M_{2, j}(A, w) \in D_{2, j}$.

Theorem 10. For any $j$ in $\{1,2,3, \ldots\}$ and for any formula $A$, $A \in I_{2, j}$ iff $A$ is $M_{2, j}$-valid.

Theorem 11. For any $j$ in $\{1,2,3, \ldots\}$ and for any formula $A, A$ is $M_{2, j}$-valid iff for any $M_{2, j}$-valuation $w$, $\xi M_{1, j}(A, w) \in \mathbf{1}_{j}$.

The following theorems 12-19 are shown with the help of the axiomatisations and semantics presented in the paper. 
TheOREM 12. Logics $I_{2,1}, I_{2,2}, I_{2,3}, \ldots I_{2, \omega}$ are simple paracomplete logics.

Theorem 13. For any $j$ and $k$ in $\{1,2,3, \ldots \omega\}$, if $j \neq k$ then $I_{2, j} \neq I_{2, k}$.

TheOREM 14. For any $j$ in $\{1,2,3, \ldots \omega\}$, the positive fragment of logic $I_{2, j}$ is equal to the positive fragment of logic $I_{2,0}$.

Theorem 15. For any $j$ in $\{1,2,3, \ldots \omega\}$, logic $I_{2, j}$ is decidable.

Theorem 16. For any $j$ in $\{1,2,3, \ldots\}$, logic $I_{2, j}$ is finitely-valued.

Theorem 17. Logic $I_{2, \omega}$ is not finitely-valued.

THEOREM 18. Logic $I_{2, \omega}$ is equal to the intersection of logics $I_{2,1}$, $I_{2,2}, I_{2,3}, \ldots$

THEOREM 19. There is a continuum of logics which include $I_{2, \omega}$ and are included in $I_{2,1}$.

\section{References}

[1] Bolotov, A., Grigoryev, O., and Shangin, V., Automated Natural Deduction for Propositional Linear-time Temporal Logic, Proceedings of the 14th International Symposium on Temporal Representation and Reasoning (Time2007), Alicante, Spain, June 28-June 30, pp.47-58, 2007.

[2] Bolotov, A .E., Shangin, V., Natural Deduction System in Paraconsistent Setting: Proof Search for PCont, Journal of Intelligent Systems, 21(1):1-24, 2012.

[3] Gentzen, G., Investigations into logical deductions, Mathematical theory of logical deduction, Nauka Publishers, M., 1967, pp. 9-74 (in Russian).

[4] Kleene, S. C., Introduction to Metamathematics, Ishi Press International, 1952.

[5] Popov, V. M., On the logic related to A. Arruda's system V1, Logic and Logical Philosophy, 7:87-90, 1999.

[6] Popov, V. M., Intervals of simple paralogics, Proceedings of the $V$ conference 'Smirnov Readings in Logic', June, 20-22, 200\%, M., 2007, pp. 35-37 (in Russian).

[7] Popov, V. M., Two sequences of simple paraconsistent logics, Logical investigations, Vol. 14, M., 2007, pp. 257-261 (in Russian). 
[8] Popov, V. M., Two sequence of simple paracomplete logics, Logic today: theory, history and applications. The proceedings of X Russian conference, June, 26-28, 2008, St.-Petersburg, SPbU Publishers, 2008, pp. 304-306 (in Russian).

[9] Popov, V. M., Semantical characterization of paracomplete logics $\mathrm{I}_{2,1}$, $\mathrm{I}_{2,2}, \mathrm{I}_{2,3}, \ldots$, Logic, methodology: actual problems and perspectives. The proceedings of conference, Rostov-on-Don, UFU Publishers, 2010, pp. 114-116 (in Russian).

[10] Popov, V. M., Sequential characterization of simple paralogics, Logical investigations, 16:205-220, 2010 (in Russian).

[11] Vasiliev, N. A., Imaginary (non-Aristotelian) logic, Vasiliev N.A. Imaginary logic. Selected works, M., Nauka Publishers, 1989, pp. 53-94 (in Russian). 